\title{
Walking along curved paths of different angles: the relationship between head and trunk turning
}

\author{
Manish N. Sreenivasa • Ilja Frissen · Jan L. Souman • \\ Marc O. Ernst
}

Received: 23 November 2007 / Accepted: 26 July 2008 / Published online: 8 August 2008

(C) The Author(s) 2008. This article is published with open access at Springerlink.com

\begin{abstract}
Walking along a curved path requires coordinated motor actions of the entire body. Here, we investigate the relationship between head and trunk movements during walking. Previous studies have found that the head systematically turns into turns before the trunk does. This has been found to occur at a constant distance rather than at a constant time before a turn. We tested whether this anticipatory head behavior is spatially invariant for turns of different angles. Head and trunk positions and orientations were measured while participants walked around obstacles in $45^{\circ}, 90^{\circ}, 135^{\circ}$ or $180^{\circ}$ turns. The radius of the turns was either imposed or left free. We found that the head started to turn into the direction of the turn at a constant distance before the obstacle $(\sim 1.1 \mathrm{~m})$ for turn angles up to $135^{\circ}$. During turns, the head was consistently oriented more into the direction of the turn than the trunk. This difference increased for larger turning angles and reached its maximum later in the turn for larger turns. Walking speeds decreased monotonically for increasing turn angles. Imposing fixed turn radii only affected the point at which the trunk started to turn into a turn. Our results support the view that anticipatory head movements during turns occur in order to gather advance visual information about the trajectory and potential obstacles.
\end{abstract}

Keywords Human locomotion · Direction change · Head orientation $\cdot$ Curved paths $\cdot$ Walking

M. N. Sreenivasa · I. Frissen · J. L. Souman · M. O. Ernst

Max Planck Institute for Biological Cybernetics,

Spemannstrasse 41, 72076 Tübingen, Germany

I. Frissen $(\square)$

Multimodal Interaction Lab, McGill University, 3459 Rue McTavish, Montreal, QC H3X 1Y1Canada

e-mail: ilja.frissen@mcgill.ca

\author{
Abbreviations \\ UCR Unconstrained radius \\ CR Constrained radius \\ CW Clockwise \\ CCW Counter-clockwise
}

\section{Introduction}

Walking along a curved path accounts for a major part of daily human locomotion. As we navigate our way through our everyday chores, our paths consist of frequent transitions from straight to curve walking and vice versa. It is indeed seldom that we find ourselves walking on a straight path over extended periods of time. This seemingly trivial task of walking along curved paths actually involves a complex interplay between the different parts of our body-the extremities, the trunk, the head and the eyes (e.g. Courtine and Schieppati 2003; Grasso et al. 1996, 1998; Imai et al. 2001; Patla et al. 1999).

Two main factors have been found to affect head movements during curve walking: stabilization and anticipatory orientation. The first one is the tendency of the head to stabilize itself by counter-acting the oscillatory yaw of the body during walking. This has been reported to be stronger during curved walking than during straight walking (Hicheur et al. 2005a; Imai et al. 2001). Another change in walking behavior during turns is a reduction in walking speed (Brogan and Johnson 2003; Hicheur and Berthoz 2005; Hicheur et al. 2005b, 2007; Imai et al. 2001; Olivier and Cretual 2007; Vieilledent et al. 2001). Although this reduction may be due to biomechanical constraints, the slower walking speed may also add to the stabilization of the head by reducing the movement frequency (Imai et al. 2001). Thus the combined effect of head counter-oscillation 
and slower walking speed results in the head providing a more stable reference frame for egocentric visual motion perception during turning (e.g. Hicheur et al. 2005a; Prévost et al. 2002).

The focus of the current study lies more on the second factor, the anticipatory head behavior. Several studies have found that the head starts to turn into the direction of the turn 0.2-0.6 s before the trunk does (Courtine and Schieppati 2003; Grasso et al. 1996, 1998; Prévost et al. 2002). It has been suggested that this happens in order to gather advance visual information about the environment and obstacles that lie in the future walking path (Grasso et al. 1996, 1998; Hicheur et al. 2005a; Hollands et al. 2001; Patla et al. 1999; Prévost et al. 2002). Prévost et al. (2002) had participants make $90^{\circ}$ turns with different walking speeds and showed that the head turns at a constant distance rather than a constant time to the corner. We will refer to this phenomenon as the spatial invariance of anticipatory head turning. A similar result was found in a study where motion was simulated in a virtual environment (Grasso et al. 2000). However, it is presently unknown whether this spatial invariance only occurs with $90^{\circ}$ turns, or generalizes to turns of different angles.

The head not only anticipates the change in walking direction prior to a turn but continues to be offset from the orientation of the trunk during a large part of the turn. The maximum offset between head orientation and walking direction is in the range of $20^{\circ}-40^{\circ}$ (e.g. Courtine and Schieppati 2003; Grasso et al. 1996, 1998; Hicheur et al. 2005a, 2007; Imai et al. 2001; Prévost et al. 2002), while that between head and trunk/pelvis orientation is $10^{\circ}-15^{\circ}$ (Hicheur et al. 2007; Solomon et al. 2006). However, since most of these studies have used turns within a restricted range around $90^{\circ}$, it is unclear whether the relationship between head and trunk orientation depends on the size of the turn. The difference between head and trunk orientation may develop in the same, stereotypical manner for different turns as it does for $90^{\circ}$ turns. Alternatively, it is possible that this relationship depends on the angle of the turn. This would be congruent with the idea that anticipatory head turning serves the purpose of gathering information about the upcoming path.

The current study had two main purposes. First, we tested to which extent spatial invariance of head anticipation is a general phenomenon, by measuring head and trunk behavior across a large range of turning angles $\left(45^{\circ}-180^{\circ}\right)$. Second, we not only studied the relationship between head and trunk behavior before a turn, but also during turns of different angles. We tested to what extent this relationship changes with turn angle. In pilot studies we observed that participants tended to change the radius of their turns depending on the turn angle. We therefore also evaluated to what extent turn radius plays a role in head-trunk interac- tions, by constraining the turn radius and comparing this to a conditions where participants were free to choose their walking path around obstacles.

\section{Methods}

Participants

A total of 24 healthy participants (11 males, 13 females: mean age $24 \pm 2.5$ years) took part in the experiment. They were paid for their participation and were naïve with respect to the purpose of the experiment. Participants had normal or corrected-to-normal vision and none reported any sensorimotor deficit that would interfere with the task. Participants gave their informed consent before taking part in the experiment, which was performed in accordance with the ethical standards laid down in the 1964 Declaration of Helsinki.

\section{Apparatus}

The experiment was conducted in the tracking lab of the Max Planck Institute for Biological Cybernetics in Tübingen. The lab is a $15 \times 12 \mathrm{~m}$ well lit empty walking space, equipped with an optical tracking system (Vicon, OMG Plc., Oxford, UK). The tracking system consists of 16 infrared cameras, which allow for an accuracy of position tracking of better than $1 \mathrm{~mm}$, and of orientation of better than $0.3^{\circ}$, in the entire walking area. Sampling rate was $90 \mathrm{~Hz}$ and all data were output in the world reference frame. The position and orientation of both the head and trunk were tracked. For head tracking, the participant wore a helmet $(<300 \mathrm{~g})$ with four infrared reflective tracking markers. Trunk was tracked by means of four infrared markers on a modified backpack that participants wore during the experiment $(2.5 \mathrm{~kg})$. Participants walked around clearly visible physical obstacles, which consisted of cardboard cylinders with a radius of $15 \mathrm{~cm}$ and height of $40 \mathrm{~cm}$.

\section{Procedure}

Participants walked along trajectories that consisted of several turns interspersed with straight parts, simulating the series of turns that occur in natural day-to-day walking. The experiment was run in a single session of approximately $45 \mathrm{~min}$, including instructions and practice trials. The experiment followed a 2 (radius: unconstrained vs. constrained) $\times 4$ (angle of turn: $45^{\circ}, 90^{\circ}, 135^{\circ}$, and $\left.180^{\circ}\right) \times 2$ [direction of turn: clockwise $(\mathrm{CW})$ or counter clockwise $(\mathrm{CCW})]$ design, with radius as a between subjects factor. Half the participants (five male, seven female) were assigned to the "unconstrained radius" condition (henceforth, UCR) while the other half (six male, six 
female) were assigned to the "constrained radius" condition (CR). In the UCR condition participants were asked to walk around the obstacles without any further instruction regarding the path to follow around the obstacle. For the CR condition, concentric circles (radius $=0.5 \mathrm{~m}$ ) were drawn around each obstacle on the ground with chalk. In this condition, participants were instructed to follow the circles during the turn without looking down at them. The withinsubject conditions were tested in random order.

On each trial participants followed pre-specified paths that were based on an $8 \times 8 \mathrm{~m}$ grid of physical obstacles. In order to test the different angles of turn, different paths through the grid were constructed (see Fig. 1). Paths consisted of both straight parts as well as a number of turns, and were constructed in such a way that they yielded either four $\mathrm{CW}$ or four CCW turns, with the exception of the $135^{\circ}$ condition which consisted of two $\mathrm{CW}$ and two $\mathrm{CCW}$ turns. The conditions $45^{\circ}, 90^{\circ}$, and $180^{\circ}$ were repeated three times for each direction and condition $135^{\circ}$ was repeated six times. This, in the end, resulted in 12 actual turns for each combination of angle and direction of turn.

Trials started with a verbal instruction as to which path was to be executed. Before actual data collection participants were familiarized with the procedure. They were shown graphical depictions of the paths and practiced the conditions.

\section{Data analysis}

All analysis of Vicon tracking data was done in Matlab 2006b (The Mathworks, USA). Raw head and trunk data were low-pass filtered (zero-phase shift Butterworth filter, cut-off frequency $10 \mathrm{~Hz}$ ) to reduce noise.

To determine the start of head or trunk turning we first calculated the standard deviation of head and trunk orientation about the yaw axis during straight walking. This was done for all the straight parts on a trial-by-trial basis and for each subject separately. Standard deviations for the straight parts were then averaged across trials and direction of turn but separately for each condition. Head anticipation of a turn was set as the point where head orientation exceeded three times the average standard deviation relative to the straight-ahead direction. The start of a turn was similarly calculated from the trunk orientation. The end of a turn was determined as the point where trunk orientation had turned to the specified angle of that condition (i.e. $45^{\circ}, 90^{\circ}, 135^{\circ}$ or $180^{\circ}$ ) minus $5^{\circ}$. Figure 2 illustrates this analysis and shows most of the parameters extracted from the data. The radius of a turn was computed as the average distance between the trajectory and the obstacle between turn start and turn end points.

In the literature on curve walking (see "Introduction") different dependent measures are used to describe head and trunk behavior. To be able to compare with relevant results from the literature we employ some of the same measures. Thus, the following six parameters were calculated:

1. Head anticipation distance with respect to obstacle.

2. Trunk anticipation distance with respect to obstacle.

3. Anticipation of the head relative to trunk.

4. Maximum relative head yaw with respect to heading.

5. Maximum relative head yaw with respect to trunk yaw.

6. Walking speed in curves and during the approach to a turn.

We determined the distance of the point where the head started to turn with respect to the obstacle as well as to the point where the trunk started to turn. Distances were computed along the walking direction of the straight part directly preceding a turn as shown in Fig. 2a. The anticipation of the head relative to the trunk (parameter 3 ) is the difference between these two measures.

In the literature, two different references for determining the maximum relative yaw of the head are used (here we use the terms orientation and yaw interchangeably). The first reference is the heading, which is calculated using consecutive head positions (e.g. Courtine and Schieppati 2003; Grasso et al. 1996, 1998; Hicheur et al. 2005a, 2007; Imai et al. 2001; Prévost et al. 2002). The second reference is the orientation of the trunk/pelvis (Hicheur et al. 2007; Solomon et al. 2006). Maximum relative yaw of the head was determined relative to these references for the period between trunk turn start and trunk turn end (Fig. 2c). The walking speed during the turns was calculated over the period between the start and end points of a turn, and walking speed during approach was the average speed from $3 \mathrm{~m}$ away to just before turn start. The straight walking part
Fig. 1 Bird's eye view of the four different walking paths for one participant. In these examples only counter-clockwise turns are shown. Dots indicate the obstacles and concentric circles represent the chalk lines used to guide participants in the constrained radius condition
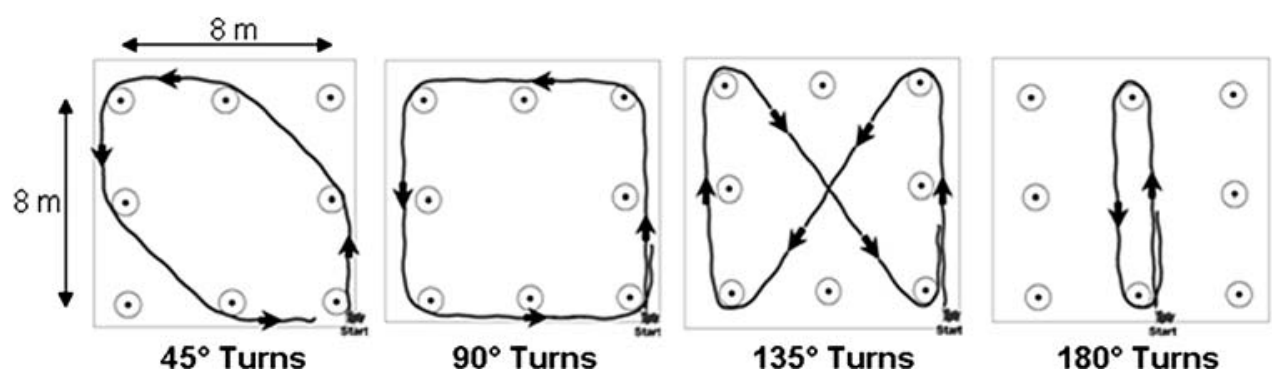

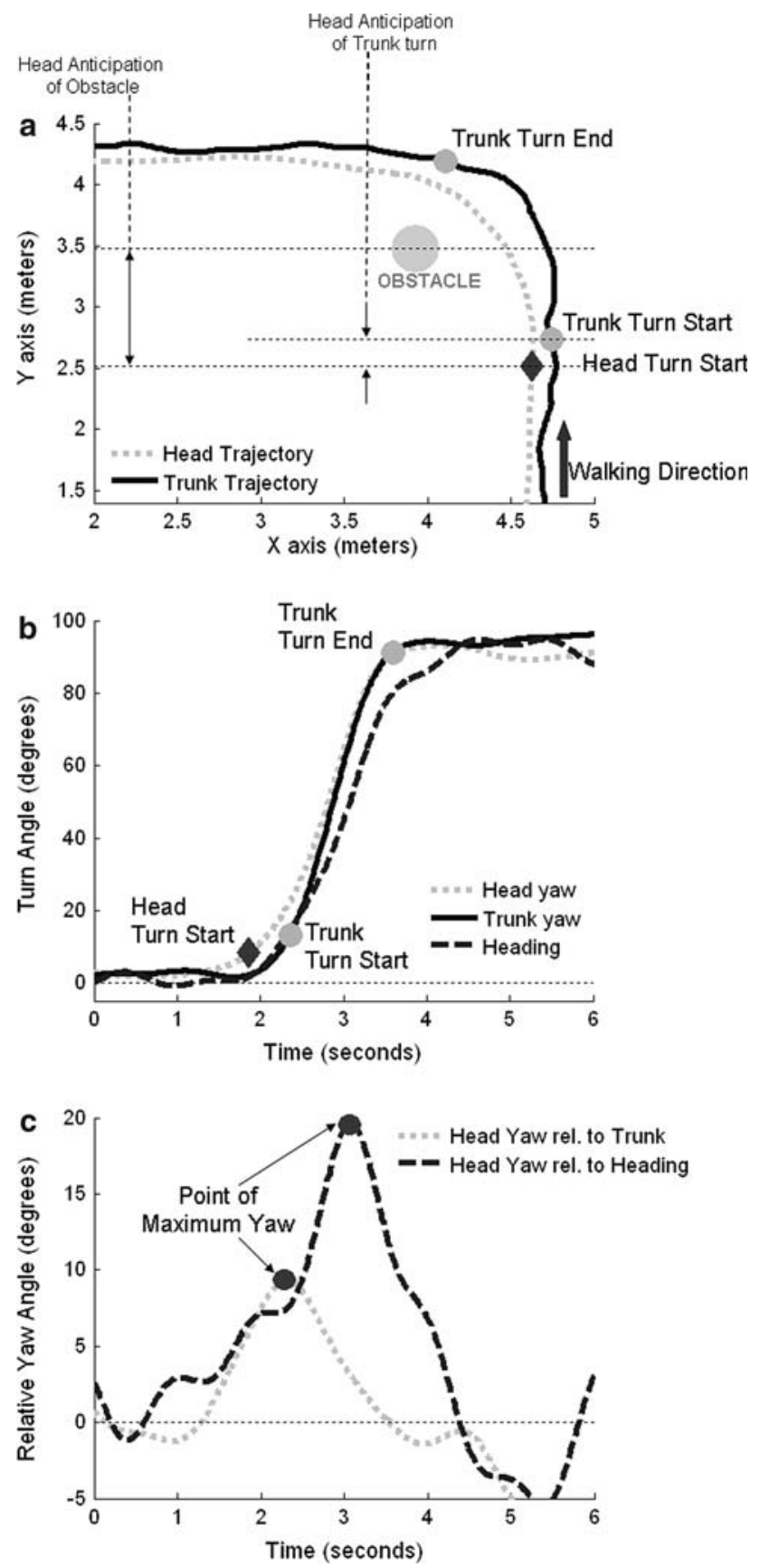

Fig. 2 Data analysis procedure. a Trajectory of head (dotted line) and trunk (solid line, offset both vertically and horizontally from head trajectory for clarity) during a sample $90^{\circ}$ turn around the obstacle (shaded circle). Grey circles on the trunk trajectory indicate start and end of turn. The diamond on the head trajectory represents the point where the head started to turn. Also illustrated are the anticipation distances of the head with respect to obstacle and trunk (arrows). b Progression of head yaw (dotted), trunk yaw (solid) and heading (dashed) over time for the same $90^{\circ}$ turn as in panel a (same symbols as used in panel a). c Relative yaw of the head with respect to heading (dashed) and trunk (dotted)

before the first turn of the $45^{\circ}$ condition paths were omitted from this analysis because they included the acceleration phase from standstill.
Mixed design ANOVAs were performed on each parameter using SPSS 12.0 (SPSS Inc., USA), with the CR versus UCR as a between-subjects factor. For all measures we collapsed the data across direction of turn. Significance level was set at 0.05 . In cases of violation of the sphericity assumption, we applied the Greenhouse-Geisser correction and report the corrected degrees of freedom. For comparing between consecutive pairs of angles of turn (i.e., $45^{\circ}$ vs. $90^{\circ}, 90^{\circ}$ vs. $135^{\circ}$ and $135^{\circ}$ vs. $180^{\circ}$ ) we used pairwise contrasts tests.

\section{Results}

Effect of constraining the radius of turns

Our manipulation of constraining the radius of turns was effective. The variability in the distance of the participants to the obstacle during turns was reduced in the CR condition. This was confirmed by a 2 (CR vs. UCR) $\times 4$ (angle of turn) repeated measures ANOVA which showed both main effects to be significant $(P$ 's $<0.001)$ as well as their interaction $(F(3,66)=16.38, P<0.001)$. Simple effects $t$-tests showed that the interaction was caused by significant reductions for $135^{\circ}$ and $180^{\circ}$ turns (25 and $46 \%$ respectively). The mean distance to the obstacle was not significantly different for the $\mathrm{CR}$ and UCR conditions $(F(1,22)=0.621, P=0.44)$, indicating that our manipulation changed the shape of the path around the obstacle, rather than the overall distance to the obstacle.

\section{Turn anticipation}

Figure 3a shows the anticipation distances of the head to the obstacle. For both UCR and CR conditions head anticipation occurred around $1.1 \mathrm{~m}$ before the obstacle for $45^{\circ}$ $135^{\circ}$ turns. In case of $180^{\circ}$ turns, the head started to turn closer $(\sim 0.9 \mathrm{~m})$ to the obstacle for both conditions. The main effect of angle of turn was significant $(F(2.6$, $57.7)=5.38, P=0.004)$ but no effect of, or interaction with, constraining the radius was found $\left(P^{\prime} \mathrm{s}>0.23\right)$. There was no difference in head anticipation distance between $45^{\circ}$ and $90^{\circ}$, or $90^{\circ}$ and $135^{\circ}$ (both $F$ 's $<1$ ), but the difference between the $135^{\circ}$ and $180^{\circ}$ conditions was significant $(F(1,22)=15.83, P=0.001)$.

A different pattern of results was found for the location where the trunk started to turn relative to the obstacle (Fig. 3b). In the CR condition, this location did not change for different turn angles, whereas for UCR turns it occurred progressively closer to the obstacle for higher turn angles. This interaction between turn angle and constraining the radius was significant $(F(2.28,50.1)=6.41, P=0.002)$. We therefore conducted separate repeated measures ANOVAs 
Fig. 3 Mean distance to the obstacle at which the head (a) and trunk (b) started to turn. Panel $c$ shows the difference between these two measures. Error bars indicate the standard errors of the mean across participants
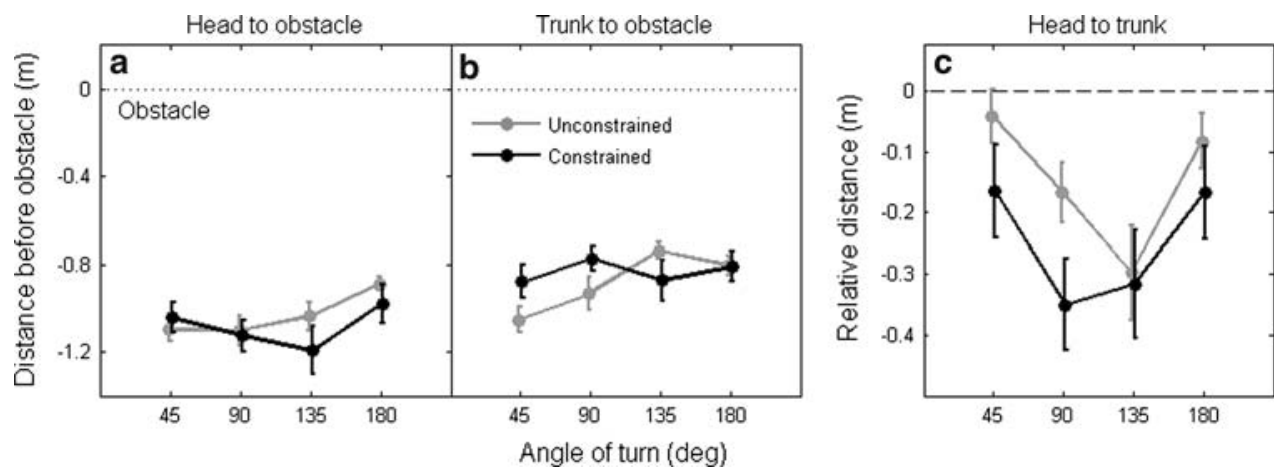

for UCR and CR. For UCR, there was a significant effect of angle of turn $(F(1.8,19.5)=8.59, P=0.003)$. Pairwise contrast tests showed that only the difference between $90^{\circ}$ and $135^{\circ}$ was significant $(F(1,11)=5.93, P=0.033)$. For $\mathrm{CR}$ the effect of angle of turn was not significant $(F(2.6$, 28.4) $=2.39, P=0.097$ ).

The anticipation distance of the head relative to the trunk (Fig. 3c), was smaller for $45^{\circ}$ and $180^{\circ}$ turns than for $90^{\circ}$ and $135^{\circ}$ turns. The main effect of turn angle was significant $(F(2.6,58.1)=13.06, P<0.001)$, with significant differences between $45^{\circ}$ and $90^{\circ}(F(1,22)=21.64, P<0.001)$, and between $135^{\circ}$ and $180^{\circ}(F(1,22)=18.98, P<0.001)$. Constraining the radius did not have a significant main effect nor did we find an interaction with angle of turn (both $P$ 's $>0.21$ ).

\section{Relative head yaw during turns}

The maximum relative yaw of the head with respect to both heading and trunk yaw is shown in Fig. 4. Irrespective of which reference was used or whether radius was constrained or not, we found an increase of relative yaw for angles up to $135^{\circ}$ and a leveling off after that. When considering the results as a function of reference type we found that these clearly lead to different estimates of the magnitude of relative yaw.

Using the heading as a reference results in relative yaws that range from $12.7^{\circ}$ for $45^{\circ}$ turns to around $40.5^{\circ}$ for $135^{\circ}$ and $180^{\circ}$ turns. Angle of turn had a significant effect on head-heading relative yaw $(F(1.5,33.6)=89.47$, $P<0.001$ ), while constraining the turn radius had no effect. Planned comparisons (collapsed across UCR and CR) showed significant differences between $45^{\circ}$ and $90^{\circ}$ $(F(1,22)=183.07, P<0.001)$, and between $90^{\circ}$ and $135^{\circ}$ $(F(1,22)=54.02, \quad P<0.001)$. There was no difference between the $135^{\circ}$ and $180^{\circ}$ angles.

With respect to the trunk, the relative yaw of the head turned out to be smaller than with the heading reference. This indicates that not only the head, but also the trunk was rotated into the turn with respect to the heading. Relative yaw ranged from $5.4^{\circ}$ for $45^{\circ}$ turns to $18.8^{\circ}$ for $135^{\circ}$ turns, dropping to $16.6^{\circ}$ for $180^{\circ}$ turns. However, the pattern of results

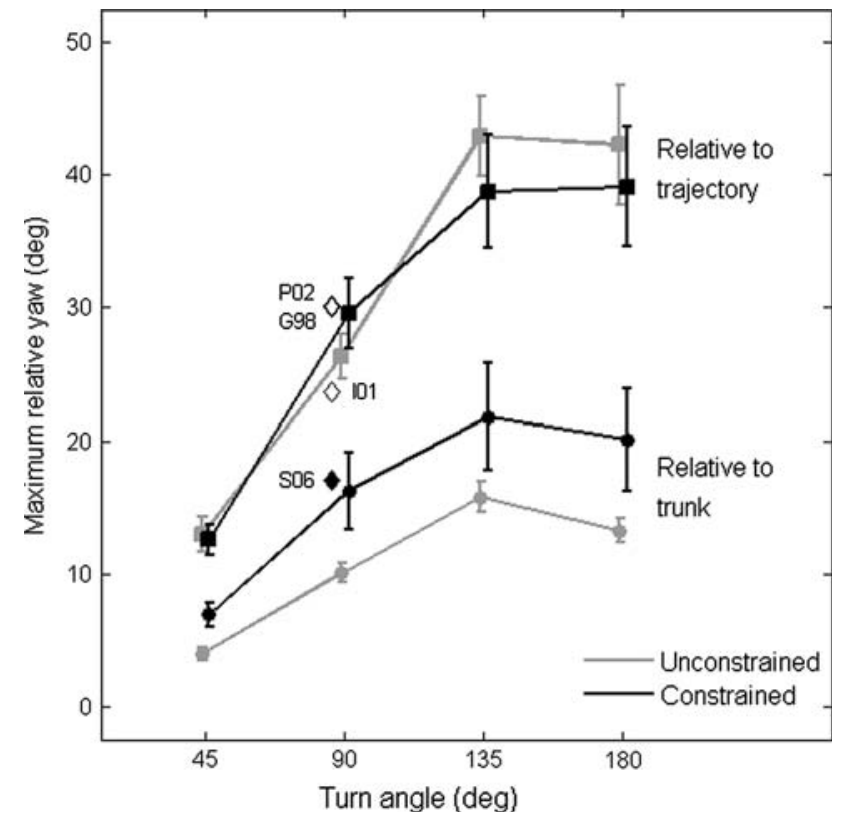

Fig. 4 Maximum relative yaw of the head with respect to heading and trunk. Error bars indicate standard errors of the mean across participants. Also shown are results from previous studies: P02: Prévost et al. 2002; G98: Grasso et al. 1998; I01: Imai et al. 2001; S06: Solomon et al. 2006. Open diamonds represent findings from studies using heading as a reference. The filled diamond represents a study using the pelvis as a reference

was the same as with the heading reference, showing an increase in relative yaw up to $135^{\circ}$ after which it leveled off. Angle of turn had a significant effect on head-trunk relative yaw $(F(1.2,27.7)=55.17, P<0.001)$. There also seemed to be a marginal effect of constraining the radius on relative yaws determined with respect to trunk $(F(1,52.7)=3.43$, $P=0.077$ ), reflecting the offset between UCR and CR. There was no interaction between angle of turn and radius constraint conditions. There were significant differences between the three subsequent angle pairs (all $P$ 's $<0.001$ ).

We also calculated the time when the maximum relative yaw (using trunk as reference) occurred during the turns relative to the start of turn. The times were $0.81,1.3,1.77$ and $1.96 \mathrm{~s}$ for $45^{\circ}, 90^{\circ}, 135^{\circ}$ and $180^{\circ}$ turns, respectively. This shows a linear increase between $45^{\circ}$ and $135^{\circ}$ turns, 
which was confirmed by linear trend analysis $(F(1,11)=$ $117.2, P<0.001)$. For $180^{\circ}$ turns, the time of occurrence of maximum relative yaw did increase but not to the same extent as for the smaller angles.

Time course of head-trunk interaction

The continuity in head-trunk interaction before and during turns is illustrated in Fig. 5. The figure shows the change in orientation of both head (solid lines) and trunk (dotted lines) over time. As can be clearly seen, the behavior of the head is highly stereotypical irrespective of turn angle. The different curves closely follow each other from before the turn only to diverge approximately $20^{\circ}$ before their respective target angles are reached. A similar stereotypical pattern was observed for the behavior of the trunk.

\section{Walking speed}

Figure 6 plots the walking speed during the approach and during turning as a function of the angle of turn. The approach speed did not depend on turn angle (mean $1.42 \pm 0.23 \mathrm{~m} / \mathrm{s}$ ). During turns, walking speed decreased across the range of angles, from 1.38 to $1.06 \mathrm{~m} / \mathrm{s}$. A 2 (phase: approach vs. turn) $\times 4$ (angle of turn) $\times 2$ (UCR vs. CR) ANOVA revealed a significant interaction between phase and angle of turn $(F(1,22)=323.64, \mathrm{p}<0.001)$, which did not depend on UCR or CR $(P>0.1)$. These results are consistent with previous studies (Brogan and Johnson 2003; Courtine and Schieppati 2003; Hicheur and Berthoz 2005; Hicheur et al. 2007).

\section{Discussion}

In this study, we investigated the effect of the angle of turn on the relationship between head and trunk orientation

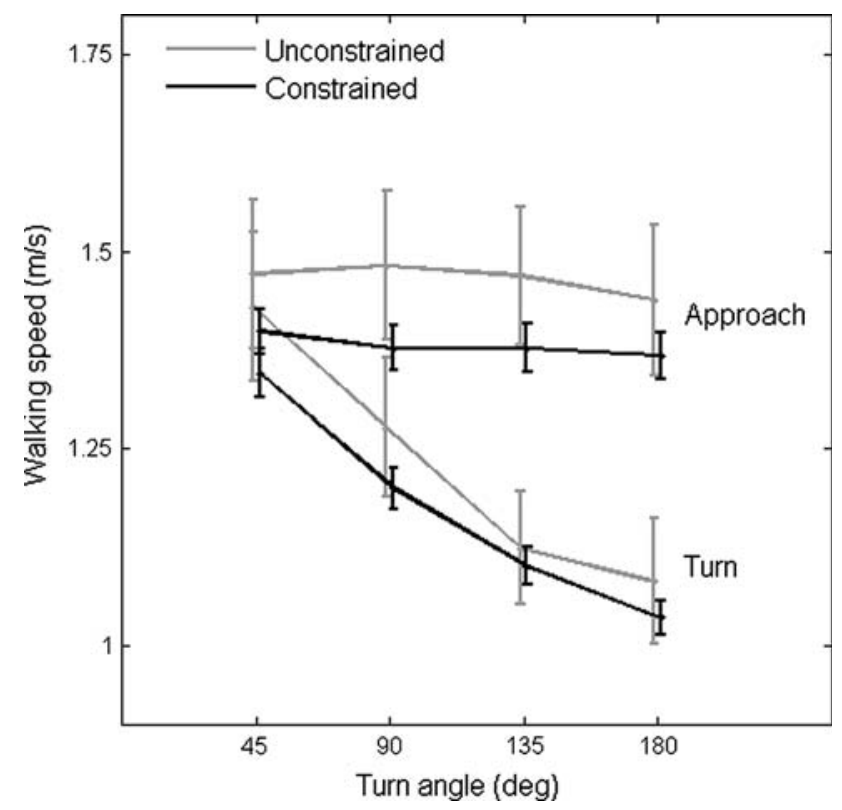

Fig. 6 Walking speed when approaching turns and during turns as a function of turn angle. Error bars indicate the standard errors of the mean across participants

during walking. There were two main findings. First, for turns between $45^{\circ}$ and $135^{\circ}$ the head started to turn at a constant distance to the obstacle $(\sim 1.1 \mathrm{~m})$. Second, the maximum relative yaw between the head and the trunk/ heading increased with angle of turn. We also observed that in this specific task (walking around an obstacle) constraining the radius of turns by drawing paths on the floor did not affect the head-trunk behavior. Only the variability in the walking trajectory and the distance to the obstacle at which the trunk started to turn differed between CR and UCR conditions. The latter would be expected since we asked subjects to use the drawn paths to initiate turns, which necessarily meant that the trunk turn occurred at the same distance for all angles.
Fig. 5 Time course of headtrunk behavior during turning. Yaw (a) and yaw rate (b) of head (solid lines) and trunk (dotted lines) over time relative to the previous straight-ahead direction. The different curves were aligned at the time of head anticipation (zero on time axis, shown by the dashed vertical line). The vertical solid lines at around $0.25 \mathrm{~s}$ indicate the time when the trunk started to turn for the respective turn angles
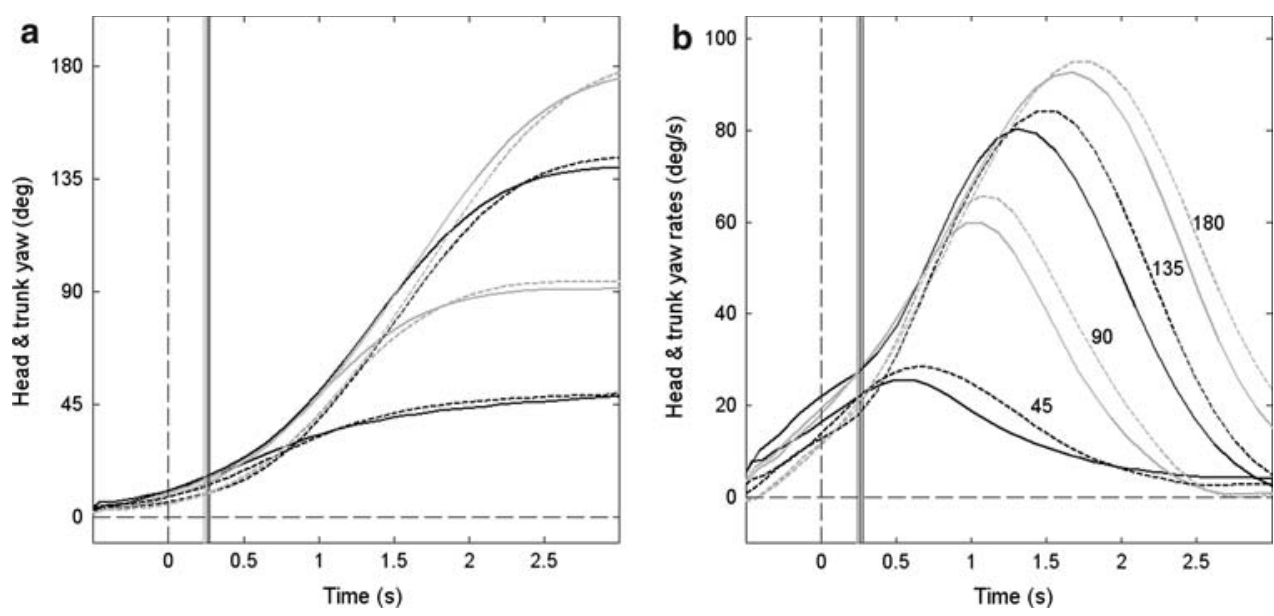
Behavioral changes during curve walking

Integrating our results with those of previous studies suggests the following account of curve walking. As one approaches and executes a turn several synergistic changes occur in the walking characteristics. The onsets of these changes precede the change in walking direction. The body center of mass is displaced in the new direction of travel through appropriate foot placement (Courtine and Schieppati 2003; Patla et al. 1991, 1999; Xu et al. 2004). This is accompanied by turning the head in the direction of the upcoming turn. Here we found that this head anticipation occurs at a constant distance from the turn (at preferred walking speeds) irrespective of turn angle. Spatial invariance has also been found in experiments using different walking speeds (Prévost et al. 2002). As the turn progresses, the trunk also starts to orient itself in the direction of turning. However, the head remains turned further into the direction of turn than the trunk. We found that the head yaw rate in this initial part of the turn is the same for different turn angles. There is also a reduction in walking speed which presumably exerts a stabilizing effect by reducing the frequency of head movements (Hicheur and Berthoz 2005; Imai et al. 2001). We found that this reduction in walking speed depends on the angle of turn. Gradually, the head yaw rate reduces as it reaches the target turn angle and the trunk aligns itself to the new direction of walking.

In the following, we split a more detailed discussion of the results into head-trunk behavior before turns and during turns.

\section{Turn anticipation}

We found that the onset of head anticipation occurred at the same distance from the obstacle across the turn angles of $45^{\circ}, 90^{\circ}$ and $135^{\circ}$. This was also true in the condition where we constrained turn radius by specifying the trajectories around the corners. This finding agrees with results from other studies (Grasso et al. 2000; Prévost et al. 2002) and extends the spatial invariance of anticipatory orienting behavior of the head to a large range of turn angles. The exception was the $180^{\circ}$ case where anticipatory distance was reduced. This could be because a complete U-turn is an extreme case and therefore caused different behavior.

Since we did not manipulate walking speeds (participants walked at their own preferred speed) the spatial invariance we found could in fact be due to a temporal invariance. However, this seems unlikely given the study by Prévost et al. (2002) which presents evidence against a temporal invariance of head anticipation. They used different walking speeds and found that anticipation occurred at different times but at a constant distance from the turn (see also Grasso et al. 2000).
A possible explanation for the spatial invariance of turn anticipation is that head turning happens at a fixed number of steps before a turn, rather than at a fixed distance. This would predict that the anticipation distance would be different for persons of different height. However, the restricted range of participant heights in our study prevents us from testing this hypothesis reliably with our current data.

Prévost et al. (2002) used a fixed yaw criterion to calculate turn anticipation distance. We used a criterion based on the variability of the head/trunk yaw during straight walking. This seems more appropriate since it takes into account differences in walking behavior between individuals as well as turn angles. Although applying the criterion used by Prévost et al. to our data produces different estimates of anticipation distance to those reported above, it does not qualitatively change our results.

\section{Relative head yaw during turns}

In contrast to the invariance of anticipatory head behavior for different turn angles, we found that the maximum relative yaw between head and heading/trunk increased with turn angle. In addition, we found that the relative yaw leveled off between turns of $135^{\circ}$ and $180^{\circ}$. This was true for both UCR and CR conditions and did not depend on the reference used (heading or trunk). One of the reasons for the leveling off could simply be a matter of comfort, where turning the head more than a certain amount becomes uncomfortable.

As illustrated in Fig. 4, the results from our study correspond well with studies that used heading as a reference (Grasso et al. 1998; Imai et al. 2001; Prévost et al. 2002) as well as with those that used the trunk/pelvis (Hicheur et al. 2007; Solomon et al. 2006). A similar trend of increasing relative yaw for turns of higher angles was also reported in a recent study by Hicheur et al. (2007) although their task and setup were very different from those in our study. In addition, we found that the time of occurrence of maximum relative yaw increased linearly for larger turn angles. These results are also consistent with those of Hicheur et al. (2007). This linear trend broke down for $180^{\circ}$ turns, for the simple reason that the maximum relative yaw for $180^{\circ}$ turns did not differ from that for $135^{\circ}$ turns.

For turns of greater angles, a larger part of the future walking path is out of view. One reason for the increase in head yaw relative to the trunk with larger angles may therefore be that the person tries to maximize the amount of available visual information about the upcoming path. Although we did not measure eye position in our study, it has been shown that changes in gaze direction are generally implemented by a combination of eye and head movements in the same direction (Barnes 1979; Guitton and Volle 1987). This is especially true for large gaze shifts (Einhäuser et al. 
2007). For example, during a simple walking task consisting of a $90^{\circ}$ turn it has been found that the eyes smoothly lead the head by looking further into the direction of turning (Imai et al. 2001). The increase in relative yaw between head and trunk with larger turn angles may well reflect a larger change in gaze direction. Our results suggest that this anticipation of the future path already starts before the actual turn and smoothly continues during the actual turn.

Taken together, these results build the basis for an understanding of the processes involved in human locomotion, which might be of interest in different application areas. For example, such results could probably serve as the ground truth to aid (early) diagnosis of pathological movements, they might guide assessment of rehabilitation, or such data might be useful for more realistic animations of virtual characters in movies or games. At least, however, these results contribute to a more thorough understanding of the processes involved in curve walking which is of obvious importance to any complete model of human locomotion.

Acknowledgments This work was supported by the European research project CyberWalk (EC-contract number FP6-511092; see http://www.cyberwalk-project.org), and by the Deutsche Forschungsgemeinschaft DFG (Sonderforschungsbereich 550).

Open Access This article is distributed under the terms of the Creative Commons Attribution Noncommercial License which permits any noncommercial use, distribution, and reproduction in any medium, provided the original author(s) and source are credited.

\section{References}

Barnes GR (1979) Vestibulo-ocular function during co-ordinated head and eye movements to acquire visual targets. J Physiol 287:127147

Brogan DC, Johnson NL (2003) Realistic human walking paths. Proceedings of Computer Animation and Social Agents (CASA), pp 94-101

Courtine G, Schieppati M (2003) Human walking along a curved path. I. Body trajectory, segment orientation and the effect of vision. Eur J Neurosci 18:177-190

Einhäuser W, Schumann F, Bardins S, Bartl K, Böning G, Schneider E, König P (2007) Human eye-head co-ordination in natural exploration. Network 18:267-297
Grasso R, Glasauer S, Takei Y, Berthoz A (1996) The predictive brain: anticipatory control of head direction for the steering of locomotion. NeuroReport 7:1170-1174

Grasso R, Prévost P, Ivanenko YP, Berthoz A (1998) Eye-head coordination for the steering of locomotion in humans: an anticipatory synergy. Neurosci Lett 253:115-118

Grasso R, Ivanenko YP, McIntyre J, Viaud-Delmon I, Berthoz A (2000) Spatial, not temporal cues drive predictive orienting movements during navigation: a virtual reality study. NeuroReport 11:775-778

Guitton D, Volle M (1987) Gaze control in humans: eye-head coordination during orienting movements to targets within and beyond the oculomotor range. J Neurophysiol 58:427-459

Hicheur H, Berthoz A (2005) How do humans turn? Head and body movements for the steering of locomotion. In: Proceedings of 2005 5th IEEE RAS international conference on humanoid robots

Hicheur H, Vieilledent S, Berthoz A (2005a) Head motion in humans alternating between straight and curved walking path: combination of stabilizing and anticipatory orienting mechanisms. Neurosci Lett 383:87-92

Hicheur H, Vieilledent S, Richardson MJE, Flash T, Berthoz A (2005b) Velocity and curvature in human locomotion along complex curved paths: a comparison with hand movements. Exp Brain Res 162:145-154

Hicheur H, Pham QC, Arechavaleta G, Laumond JP, Berthoz A (2007) The formation of trajectories during goal-oriented locomotion in humans I. A stereotyped behavior. Eur J Neurosci 26:2376-2390

Hollands MA, Sorensen KL, Patla AE (2001) Effects of head immobilization on the coordination and control of head and body reorientation and translation during steering. Exp Brain Res 140:223-233

Imai T, Moore ST, Raphan T, Cohen B (2001) Interaction of the body, head, and eyes during walking and turning. Exp Brain Res 136:1-18

Olivier AH, Cretual A (2007) Velocity/curvature relations along a single turn in human locomotion. Neurosci Lett 412:148-153

Patla AE, Prentice SD, Robinson C, Neufeld J (1991) Visual control of locomotion: strategies for changing direction and for going over obstacles. J Exp Psychol Hum Percept Perform 17:603-634

Patla AE, Adkin A, Ballard T (1999) Online steering: coordination and control of body center of mass, head and body reorientation. Exp Brain Res 129:629-634

Prévost P, Ivanenko Y, Grasso R, Berthoz A (2002) Spatial invariance in anticipatory orienting behavior during human navigation. Neurosci Lett 339:243-247

Solomon D, Kumar V, Jenkins RA, Jewell J (2006) Head control strategies during whole-body turns. Exp Brain Res 173:475-486

Vieilledent S, Kerlirzin Y, Dalbera S, Berthoz A (2001) Relationship between velocity and curvature of a human locomotor trajectory. Neurosci Lett 305:65-69

Xu D, Carlton LG, Rosengren KS (2004) Anticipatory postural adjustments for altering direction during walking. J Mot Behav 36:316326 